was a secondary progressive decline to a lower plateau of $+8.0 \pm 1.8 \mathrm{~mm} \mathrm{Hg}(\mathrm{p}=0.004)$, Abstract 004 figure 1. The initial increment was caused by an immediate rise in flow by $+9.1 \pm 2.4 \%$ $(p=0.007)$ which did not drop later. The secondary decline in pressure was caused by a delayed gradual decline in total peripheral resistance. Finometer-derived non-invasive blood pressure tracked invasive pressure closely $(\mathrm{r}=0.97)$.

Conclusion When AV delay is made more favourable, only the instant pressure increment is caused by increase in stroke volume. The secondary pressure decline is caused by systemic vasodilatation. Design of AV optimisation protocols, which face severe challenge of signal vs noise, might benefit from recognition that not all beats are equally informative: the first few after a transition are most signal-rich.

\section{CARDIAC MRI: UNDERSTANDING MYOCARDIAL MOTION TO PREDICT REMODELLING PRE CARDIAC RESYNCHRONISATION THERAPY}

doi:10.1136/heartjnl-2012-301877b.5

${ }^{1} \mathrm{~S}$ G Duckett, ${ }^{*} \mathrm{~W}$ Shi, ${ }^{2} \mathrm{X}$ Zhuang, ${ }^{1} \mathrm{~A}$ Shetty, ${ }^{3} \mathrm{M}$ Ginks, ${ }^{3} \mathrm{C}$ A Rinaldi, ${ }^{3} \mathrm{G}$ Carr-White, ${ }^{2} \mathrm{D}$ Rueckert, ${ }^{1} \mathrm{R} S \mathrm{R}$ Razavi. ${ }^{1}$ Kings College London, London, UK; ${ }^{2}$ Imperial College London, London, UK; ${ }^{3}$ Guy's and St Thomas' Hospital, London, UK

Introduction A significant number of patients undergoing Cardiac Resynchronisation Therapy (CRT) do not remodel. Assessing global dyssynchrony has the potential to improve patient selection. We developed a framework for comparing measures of myocardial motion from cardiac magnetic resonance (CMR) imaging and evaluated the potential of these techniques to improve patient selection. Methods 48 patients recruited, (43 males, 63.8 \pm 13.9 years), NYHA class $2.9 \pm 0.5$, ejection fraction $25 \pm 9 \%$. Patients had LBBB (ORS $154 \pm 24 \mathrm{~ms}$ ). Acute haemodynamic response was measured at time of implant with a pressure wire in the LV measuring change in $\mathrm{dP} /$ $\mathrm{dt}_{\max } . \mathrm{A}>10 \%$ increase in $\mathrm{LV}-\mathrm{dP} / \mathrm{dt}_{\max }$ from baseline was considered an acute response. Decrease in end systolic volume (ESV) $\geq 15 \%$ at 6 months was used to determine remodelling. CMR was performed prior to CRT. A novel framework was developed. Key steps included: (1) detection of heart and myocardium segmentation from anatomical CMR cine images; (2) detection of endo and epi-cardial surfaces for wall thickening computation; (3) extraction of deformation fields within the myocardium for strain computation. A systolic dyssynchrony index (SDI) was produced for all parameters
Acute Response
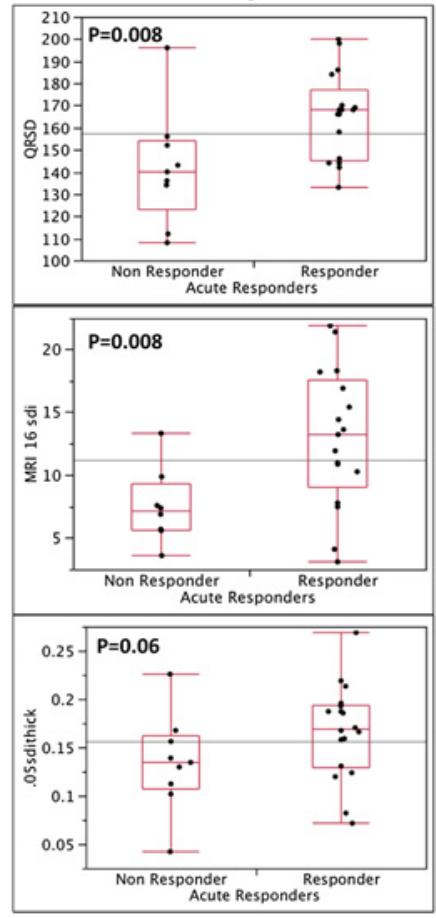

Abstract 005 Figure 1 Shows the ANOVA plots for acute response and remodelling for QRS duration, volume and muscle thickening derived SDI.

which included volume change, muscle thickening, radial, circumferential, longitudinal strain and combined strain. High SDI denoted dyssynchrony. Results Pre-implant ESV $175 \pm 64 \mathrm{ml}$, post-implant ESV $155 \pm 68 \mathrm{ml}(\mathrm{p}<0.01) .20$ (44\%) patients remodelled. We found a strong relationship between volume derived SDI and acute response $(p=0.008)$ and remodelling $(p<0.001)$ (Abstract 005 figure 1$)$. We found a weaker relationship with remodelling and muscle thickening SDI $(p=0.001)$ and no relationship with a SDI derived from strain indexes (Abstract 005 figure 2). Volume SDI $\geq 10 \%$ was highly sensitive (0.94) and specific (0.87) for predicting remodelling. Volume SDI was far superior for predicting remodelling than any other method. The intra-observer average difference for volume SDI

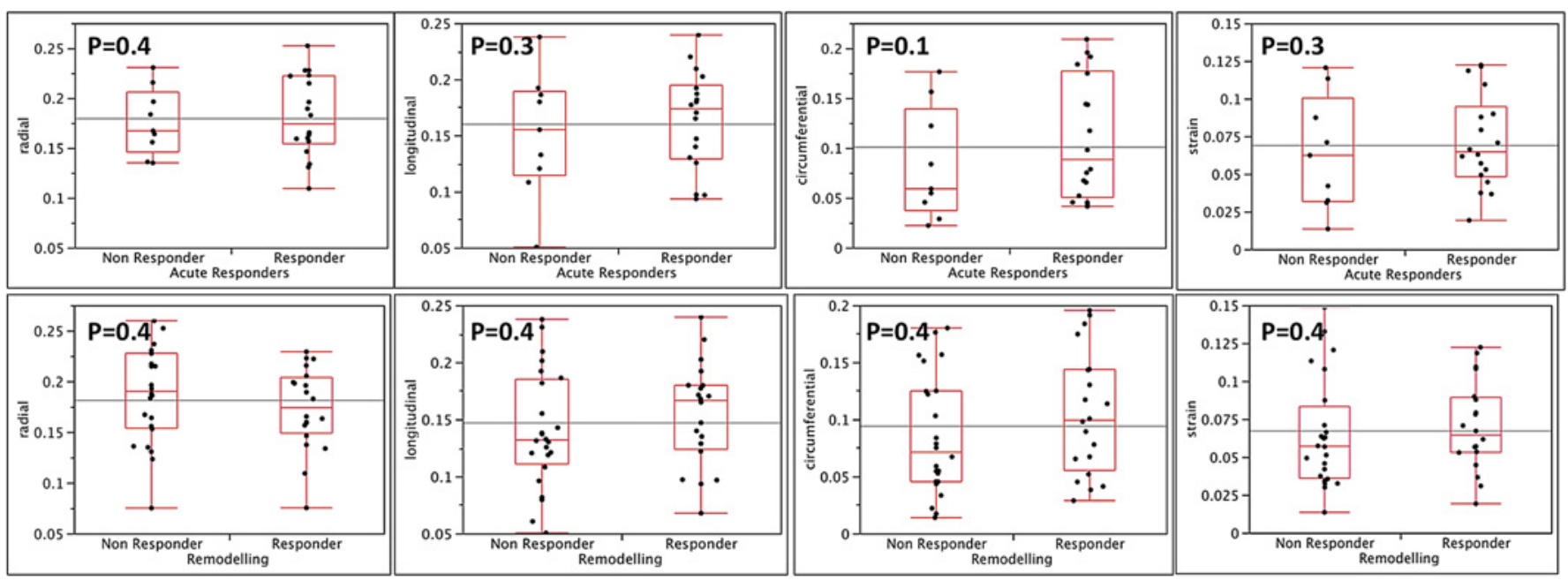

Abstract 005 Figure 2 Shows the ANOVA plots for acute response (top row) and remodelling (bottom row) for SDI derived from various types of strain. 
was $0.04 \pm 0.3 \%$ and $\mathrm{COV}$ was $1.8 \pm 1.2 \%$ and the inter-observer average difference was $0.55 \pm 1.4 \%$ and COV was $4.2 \pm 4.6 \%$.

Conclusion A volume derived SDI from cine CMR strongly predicts remodelling post CRT. It is a highly reproducible measurement that has significant potential clinical implications in the future.

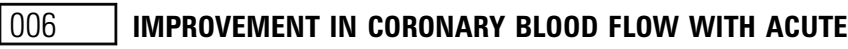 BIVENTRICULAR PACING IS PREDOMINANTLY DUE TO AN INCREASE IN A DIASTOLIC BACKWARD-TRAVELLING DECOMPRESSION (SUCTION) WAVE}

doi:10.1136/heartjnl-2012-301877b.6

A Kyriacou, * Z I Whinnett, S Sen, P Pabari, D W Davies, N S Peters, P Kanagaratnam, J Mayet, A D Hughes, D P Francis, J E Davies. NHLI, St Mary's Hospital, Imperial College Healthcare NHS Trust, London, UK

Background Normal coronary blood flow is principally determined by a backward-travelling decompression (suction) wave in diastole. This wave may be attenuated in chronic heart failure, reducing blood flow, as regional relaxation and contraction overlap in timing. We hypothesised that biventricular pacing by restoring left ventricular (LV) synchronisation and improving LV relaxation might increase this decompression wave, improving coronary flow.

Method and Results Ten CHF patients ( 9 males; age $65 \pm 12$; EF $26 \pm 7 \%$ ) with left bundle branch block (LBBB, ORS duration $174 \pm 18 \mathrm{~ms}$ ) were atrio-biventricularly paced at $100 \mathrm{bpm}$. LV pressure was measured and wave intensity calculated from invasive coronary flow velocity and pressure, with native conduction (LBBB)

Abstract 006 Table 1 Cumulative wave intensity of major left coronary artery waves and comparison of these between all four pacing states. Data are presented as mean \pm SEM

\begin{tabular}{|c|c|c|c|c|c|c|}
\hline $\begin{array}{l}\text { Wave Intensity } \\
\text { (AUC) } \times 10^{3} \\
\mathrm{~W} / \mathrm{m}^{2} / \mathrm{s}\end{array}$ & LBBB & Biv-40 & BiV-120 & BiV-OPt & $\begin{array}{l}\text { p Value } \\
\text { (BiV-0pt } \\
\text { vs LBBB) }\end{array}$ & $\begin{array}{l}\text { p Value } \\
\text { (Biv-40 } \\
\text { vs LBBB }\end{array}$ \\
\hline \multicolumn{7}{|l|}{ Systole } \\
\hline $\begin{array}{l}\text { 1. Forward } \\
\text { compression }\end{array}$ & $9.1 \pm 1.9$ & $8.6 \pm 1.9$ & $11.4 \pm 2.3$ & $12.1 \pm 2.5$ & 0.04 & 0.51 \\
\hline $\begin{array}{l}\text { 2. Backward } \\
\text { compression }\end{array}$ & $7.5 \pm 1.5$ & $7.2 \pm 1.7$ & $9.0 \pm 1.6$ & $9.9 \pm 1.9$ & 0.01 & 0.75 \\
\hline $\begin{array}{l}\text { Net wave } \\
\text { contribution to } \\
\text { forward flow } \\
\text { (1 minus } 2 \text { ) }\end{array}$ & $1.6 \pm 1.4$ & $1.4 \pm 1.0$ & $2.4 \pm 1.3$ & $2.3 \pm 1.7$ & & \\
\hline \multicolumn{7}{|l|}{ Diastole } \\
\hline $\begin{array}{l}\text { 3. Forward } \\
\text { decompression }\end{array}$ & $2.1 \pm 0.5$ & $3.2 \pm 0.7$ & $3.5 \pm 0.8$ & $3.3 \pm 0.6$ & 0.15 & 0.20 \\
\hline $\begin{array}{l}\text { 4. Late forward } \\
\text { compression } \\
\text { (suction) }\end{array}$ & $3.2 \pm 0.8$ & $2.4 \pm 0.9$ & $2.7 \pm 0.6$ & $2.8 \pm 0.9$ & 0.76 & 0.42 \\
\hline $\begin{array}{l}\text { 5. Backward } \\
\text { decompression } \\
\text { (suction) }\end{array}$ & $7.8 \pm 1.4$ & $7.6 \pm 1.3$ & $10.1 \pm 2.0$ & $10.4 \pm 2.1$ & 0.01 & 0.71 \\
\hline \multirow[t]{2}{*}{$\begin{array}{l}\text { Net wave } \\
\text { contribution } \\
\text { to forward } \\
\text { flow ( } 5 \text { plus } \\
4 \text { minus } 3 \text { ) }\end{array}$} & $8.8 \pm 1.9$ & $6.7 \pm 1.8$ & $9.3 \pm 2.4$ & $9.9 \pm 2.1$ & & \\
\hline & & & & & $p$ Value & \\
\hline $\begin{array}{l}\text { Proportion of } \\
\text { diastolic } \\
\text { contribution } \\
\text { to total } \\
\text { net wave } \\
\text { contribution } \\
\text { to forward flow }\end{array}$ & $85 \pm 5 \%$ & $83 \pm 4 \%$ & $80 \pm 6 \%$ & $81 \pm 5 \%$ & 0.62 & \\
\hline
\end{tabular}
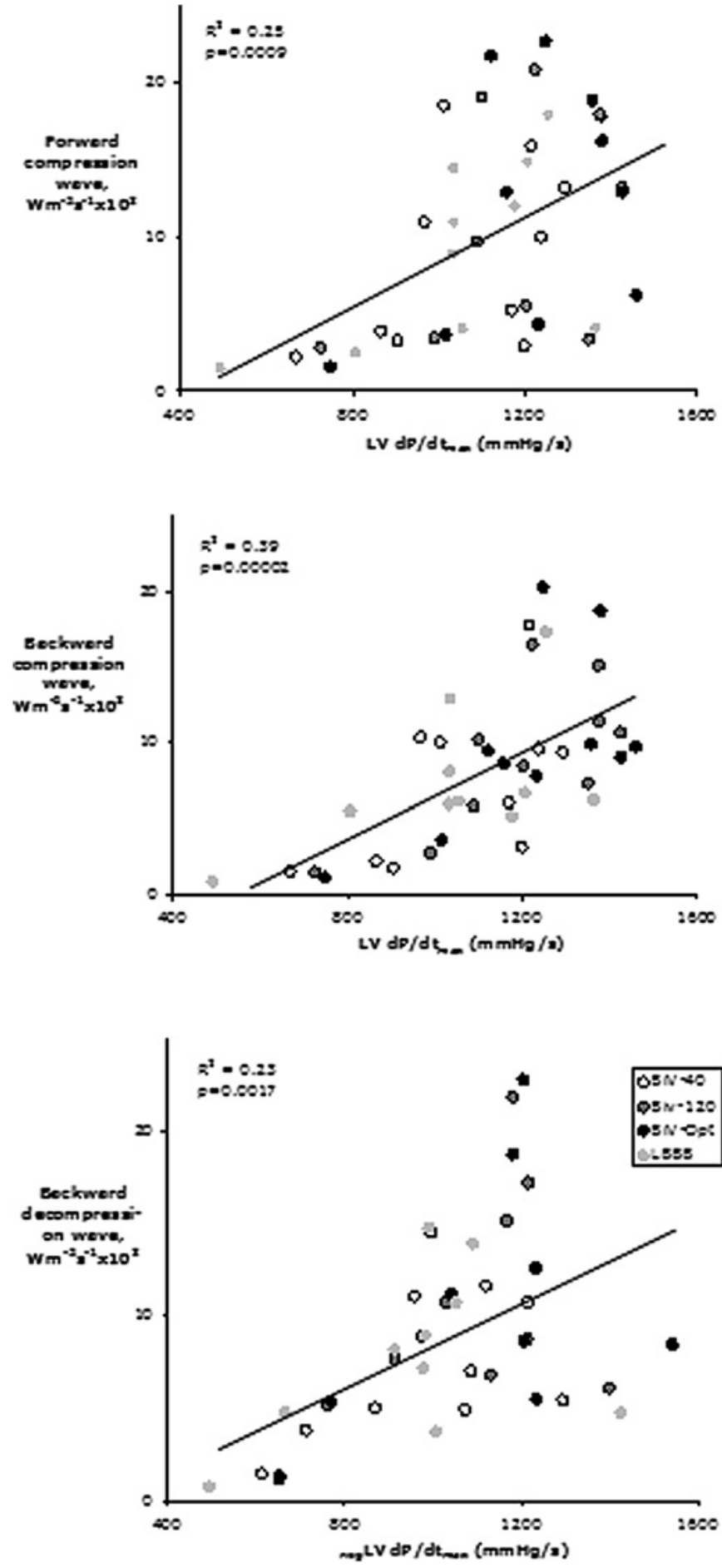

Abstract 006 Figure 1 Impact of $L V d p / d t_{\max }$ and $L V{ }_{\text {neg }} d p / \mathrm{dt}_{\max }$ on their temporally corresponding coronary waves. In each part of the cardiac cycle, the peak rate of intraventricular pressure change (LV dp/ $\mathrm{dt}_{\max }$ for systole, and LV negdP/dt $\mathrm{dt}_{\max }$ for diastole) correlated with the corresponding waves, consistent with myocardial compression and decompression of the coronary microcirculation generating the waves.

and during biventricular pacing at atrioventricular (AV) delays of $40 \mathrm{~ms}$ (BiV-40), $120 \mathrm{~ms}$ (BiV-120), and separately pre-identified haemodynamically-optimal AV delay (BiV-Opt). Compared against LBBB, BiV-Opt enhanced coronary flow by $15 \pm 4 \%(p=0.003), L V$ $\mathrm{dP} / \mathrm{dt}_{\max }$ by $19 \pm 4 \% \quad(\mathrm{p}<0.001)$ and $n e g d P / \mathrm{dt}_{\max }$ by $16 \pm 3 \%$ $(p<0.001)$. The cumulative intensity of the diastolic backward decompression (suction) wave increased by $36 \pm 8 \%(p=0.011)$. The 